\title{
Análisis de la operación del horno alto mediante la exergía térmica
}

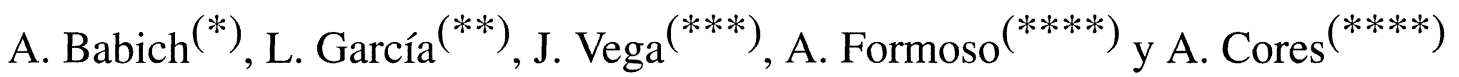

Resumen Para conocer el estado térmico del horno alto se puede utilizar la exergía térmica, que considera el nivel de temperaturas del proceso. El análisis de la magnitud de la exergía térmica, para la operación del horno alto, utilizando inyecciones por toberas de carbón pulverizado, de gas natural y viento enriquecido en oxígeno, en ambos casos muestra que la inyección de gas natural es menos efectiva que la inyección simultánea de carbón pulverizado y viento enriquecido con oxígeno.

Palabras clave: Exergía térmica. Inyección por tobera. Carbón pulverizado. Gas natural. Horno alto.

\section{Blast furnace operation analysis by thermic exergy}

\begin{abstract}
In order to know the blast furnace thermic state, the thermic exergy can be used, which considers the temperatures level of the process. The magnitude analysis of thermic exergy for blast furnace operation using pulverized coal, natural gas and oxigen injection by tuyeres, shows that natural gas injection is less effective than pulverized coal and enriched blast with oxygen simultaneous injection.
\end{abstract}

Keywords: Thermic exergy. Tuyere injection. Pulverized coal. Natural gas. Blast furnace.

\section{INTRODUCCIÓN}

Un esfuerzo considerable de los siderúrgicos está destinado a la disminución del consumo energético del horno alto y a la sustitución del coque por combustibles auxiliares. La efectividad de las diferentes variantes de utilización de combustibles auxiliares y de intensificadores de la combustión, se puede valorar mediante el balance termodinámico basado en la utilización de la exergía (1-3).

En el presente trabajo se analiza como varía la operación del horno alto cuando se inyectan combustibles por toberas mediante balances de la exergía.

(*) Univ. Estatal Tecnológica de Donestk. Donestk (Ucrania).

(**) Unión de Empresas de Recuperación de Materias Primas. La Habana (Cuba).

(***) Aceralia Corporación Siderúrgica. Gijón (Asturias, España).

(****) Centro Nacional de Investigaciones Metalúrgicas (CENIM). Avda. de Gregorio del Amo, 8. 28029-Madrid (España).

\section{EXERGÍA}

La exergía mide el máximo trabajo mecánico útil que puede realizar un sistema termodinámico. En otras palabras, la exergía permite estimar el valor del calor con diferente potencial de temperatura. El concepto de energía está relacionado con el primer principio de la termodinámica. El concepto de la exergía está relacionado con el segundo principio. Esta magnitud depende de la composición de los componentes del sistema, temperatura y presiones. El análisis exérgico de las transformaciones que ocurren en el sistema tiene en cuenta, de forma integral, la influencia de todos los parámetros de los cuales depende la generación de trabajo útil. En esto se resume la gran cantidad de información que ofrecen los métodos exérgicos para analizar los distintos procesos tecnológicos, incluido el del horno alto, desde un punto de vista energético unificado. Se considera posible un conocimiento seguro del estado térmico del horno alto y el control efectivo del mismo cuando los parámetros que lo caracterizan (temperatura del arrabio y contenido de silicio) estén unidos en un solo parámetro complejo. Por ello, no es útil emplear la entalpía debido a que una misma cantidad de calor tiene diferente 
valoración según los distintos potenciales caloríficos. En este caso es preferible usar la exergía térmica $\left(E_{t}\right)$ que considera el nivel de temperaturas del proceso (4).

Hay diferentes clases de exergía. Sin embargo, la exergía térmica se usa básicamente. Esta magnitud está constituida por las exergías química y física de la sustancia, según la ecuación:

$$
E_{t}=E_{q}+E_{f}
$$

La exergía física del arrabio se calcula por la ecuación (5):

$$
\begin{gathered}
E_{f}=C_{1}\left(T_{f}-T_{0}\right)+Q+C_{2}\left(T_{a}-T_{f}\right)- \\
-T_{0}\left(C_{1} \ln \frac{T_{f}}{T_{0}}+\frac{Q}{T_{f}}+C_{2} \ln \frac{T_{a}}{T_{f}}\right)
\end{gathered}
$$

donde: $E_{f}=$ Exergía física, $\mathrm{kJ}$.

$C_{1}=$ Capacidad calorífica del arrabio en el intervalo $T_{0}-T_{\mathrm{f}}, \mathrm{kJ} / \mathrm{kg} \cdot \mathrm{K}$.

$C_{2}=$ Capacidad calorífica del arrabio en el intervalo $T_{\mathrm{f}}-T_{\mathrm{a}}, \mathrm{kJ} / \mathrm{kg} \cdot \mathrm{K}$.

$T_{a}=$ Temperatura del arrabio, $\mathrm{K}$.

$T_{0}=$ Temperatura ambiente, $\mathrm{K}$.

$T_{f}=$ Temperatura del arrabio en la colada, $\mathrm{K}$.

$Q=$ Calor de fusión del arrabio, $\mathrm{kJ} / \mathrm{kg}$.

La exergía química del arrabio se determina mediante la ecuación:

$$
E_{q}=\Sigma Q_{i} \cdot e x_{i}
$$

donde: $E_{q}=$ Exergía química, $\mathrm{kJ}$.

$e x_{i}=$ Exergía química específica de $i$

componentes del arrabio, $\mathrm{kJ} / \mathrm{kg}$.

$Q_{i}=$ Contenido en peso de $i$ componentes

en el arrabio, $\mathrm{kg}$.

Se han calculado las exergías del estado térmico del crisol del horno alto mediante la utilización de estas ecuaciones (6).

\section{REALIZACIÓN DE BALANCES TERMODINÁMICOS MEDIANTE LA EXERGÍA}

A continuación, se analizan los balances exérgicos realizados en el horno alto para las condiciones de operación que se muestran en la tabla I.

Se estudia la operación del horno con variaciones de caudal de carbón pulverizado de 0 a 190 $\mathrm{kg} / \mathrm{ta}$, de gas natural de 0 a $175 \mathrm{~m}^{3} / \mathrm{ta}$ y del contenido de oxígeno en el viento de 21 a $33 \%$. El carbón utilizado tiene $10-12 \%$ de volátiles, $70-74 \%$ de C
TABLA I.- Índices de operación del horno alto

TABLE I.- Blast furnace operation parameters

$\begin{array}{lc}\text { Productividad, } \mathrm{t} / 24 \mathrm{~h} & 1.750 \\ \text { Consumo de coque, } \mathrm{kg} / \mathrm{ta} & 460 \\ \text { Caudal de gas natural, m} \mathrm{m}^{3} \mathrm{ta} & 84 \\ \text { Carbón vegetal pulverizado, kg/ta } & 77 \\ \text { Viento: } & \\ \text { - Caudal, } \mathrm{m}^{3} / \mathrm{min} & 1.984 \\ \text { - Temperatura, }{ }^{\circ} \mathrm{C} & 1.102 \\ \text { - Contenido de oxígeno, \% } & 24 \\ \text { - Humedad, \% } & 8 \\ \text { Composición del arrabio, \%: } & \\ \text { - Si } & 0,77 \\ \text { - Mn } & 0,88 \\ \text { - C } & 4,25 \\ \text { - S } & 0,029 \\ \text { - P } & 0,068 \\ \text { Temperatura del arrabio, }{ }^{\circ} \mathrm{C} & 1.450 \\ \text { Consumo de mineral, kg/ta } & 1.827 \\ \text { Volumen de escoria, kg/ta } & 457 \\ \text { Grado de oxidación del hierro en } & \\ \text { la carga, \% } & 16,0 \\ \text { Contenido de C en el coque, \% } & 87,6 \\ \text { Humedad del coque, } \% & 3,30 \\ \text { Grado de utilización de la capacidad } & \\ \text { reductora del CO, \% } & 38,8\end{array}$

fijo y 1,3-1,5 \% de S. Los resultados de los cálculos realizados en microordenador se muestran en las tabla II, III y IV. La información de los balances realizados se analiza mediante los coeficientes exérgicos calculados. Estos son:

- Coeficiente $K_{p}$, que determina la perfección termodinámica del horno alto, o sea, la relación entre la exergía consumida en el horno y la cantidad total de exergía suministrada.

- Coeficiente tecnológico de trabajo útil $K_{t}$, o sea, la relación de las exergías física y química del producto (arrabio), y la exergía suministrada.

- Coeficiente general de trabajo útil en la producción de arrabio $K_{g}$, o sea, la relación de las exergías física y química del arrabio más la exergía química del gas de tragante, que se envía a los consumidores externos, y la exergía suministrada.

\subsection{Inyección de carbón pulverizado (ICP)}

Con la ICP por toberas en el horno al aumentar el caudal en $10 \mathrm{~kg} / \mathrm{ta}$ ocurre un disminución de la 
TABLA II.- Balances exérgicos de la operación del horno alto con ICP

TABLE II.-Exergy balances in the blast furnace operation with PCI

\begin{tabular}{|c|c|c|c|c|c|c|}
\hline \multirow{2}{*}{ Índices } & \multirow{2}{*}{ Unidades } & \multicolumn{5}{|c|}{ Caudal ICP, $\mathrm{kg} / \mathrm{ta}$} \\
\hline & & 0 & 60 & 130 & 160 & 190 \\
\hline Consumo de coque seco & $\mathrm{kg} / \mathrm{ta}$ & 597,7 & 529,1 & 460,0 & 426,4 & 392,2 \\
\hline Consumo de $\mathrm{C}$ del coque, quemado en el viento seco & $\mathrm{kg} / \mathrm{ta}$ & 348,1 & 292,9 & 238,1 & 210,8 & 183,6 \\
\hline Volumen gas de tragante (seco) & $\mathrm{m}^{3} / \mathrm{ta}$ & $1.923,5$ & $1.967,9$ & $2.010,5$ & $2.031,0$ & $2.051,1$ \\
\hline Pérdidas de energía en el horno & $\mathrm{kg}$ ce/ta & 359,8 & 348,2 & 336,9 & 331,3 & 325,8 \\
\hline \multicolumn{7}{|l|}{ BALANCE EXÉRGICO } \\
\hline \multicolumn{7}{|l|}{ • Entradas: } \\
\hline - Exergía física del viento & $\mathrm{MJ} / \mathrm{ta}$ & $1.328,0$ & $1.335,7$ & $1.337,2$ & $1.335,6$ & $1.332,5$ \\
\hline - Exergía química del coque & $\mathrm{MJ} / \mathrm{ta}$ & $17.221,5$ & $15.243,9$ & $13,269,8$ & $12.284,1$ & $11.299,3$ \\
\hline - Exergía química de los materiales férreos & $\mathrm{MJ} / \mathrm{ta}$ & 918,1 & 918,1 & 918,1 & 918,1 & 918,1 \\
\hline - Exergía química del carbono & $\mathrm{MJ} / \mathrm{ta}$ & 0 & $2.106,6$ & $4.188,2$ & $5.228,3$ & $6.267,9$ \\
\hline Total & $\mathrm{MJ} / \mathrm{ta}$ & $19,490,6$ & $19,604,6$ & $19.713,3$ & $19,766,1$ & $19,817,8$ \\
\hline \multicolumn{7}{|l|}{ • Consumos } \\
\hline \multicolumn{7}{|l|}{ - Exergía del arrabio: } \\
\hline Física & $\mathrm{MJ} / \mathrm{ta}$ & 754,6 & 754,6 & 754,6 & 754,6 & 754,6 \\
\hline Química & $\mathrm{MJ} / \mathrm{ta}$ & $7.957,9$ & $7.957,9$ & $7.957,9$ & $7.957,9$ & $7.957,9$ \\
\hline \multicolumn{7}{|l|}{ - Exergía de la escoria: } \\
\hline Física & $\mathrm{MJ} / \mathrm{ta}$ & 533,2 & 683,2 & 691,3 & 710,8 & 767,3 \\
\hline Química & $\mathrm{MJ} / \mathrm{ta}$ & $6.366,4$ & $6.820,7$ & $7.281,1$ & $7.513,5$ & $7.747,4$ \\
\hline Total & & $16.703,3$ & $17.207,6$ & $17.776,1$ & $18.027,9$ & $18.318,3$ \\
\hline - Pérdidas exérgicas & $\mathrm{MJ} / \mathrm{ta}$ & $2.764,3$ & $2.396,7$ & $1.937,2$ & $1.738,2$ & $1.499,5$ \\
\hline - Perfección termodinámica del horno alto & $\%$ & 85,75 & 87,77 & 90,02 & 91,21 & 92,43 \\
\hline$\cdot K_{t}$ & $\%$ & 44,7 & 44,4 & 44,2 & 44,1 & 44,0 \\
\hline • $K_{g}$ & $\%$ & 77,4 & 79,2 & 81,1 & 82,1 & 83,1 \\
\hline
\end{tabular}

pérdida de energía suministrada de $2 \mathrm{~kg}$ ce/t (economía de combustible), y la exergía entrante crece en este caso en $20 \mathrm{MJ} / \mathrm{t}$. El combustible efectivo (ce) equivale a $7.000 \mathrm{kcal} / \mathrm{kg}$. Esto se explica, fundamentalmente, con el aumento de la exergía del CP utilizado en comparación con el coque sustituido.

Con la ICP ocurre un aumento en el volumen de gas de tragante y de su temperatura, creciendo a su vez la exergía de los productos de fusión. Por tanto, la suma de las pérdidas exérgicas disminuye y el proceso, al aumentar el caudal de ICP, se hace más estable termodinámicamente. En este caso crece notablemente el $K_{g}$ del horno, que a su vez determina la economía relativa (energética) de la fusión.
La ICP ocasiona cierta disminución del $K_{t}$, ya que al mantenerse constante la exergía del arrabio aumenta la entrada de exergía debido a la exergía más alta del $\mathrm{CP}$ en comparación con el coque.

\subsection{Inyección de gas natural (IGN)}

Al utilizar IGN por toberas el $K_{t}$ disminuye más rápidamente que al utilizar ICP. Sin embargo, esto ocurre en base al aumento del consumo de energía en $0,8 \mathrm{~kg} / \mathrm{t}$ de combustible estimado por cada $10 \mathrm{~m}^{3}$ de GN inyectado. La exergía de entrada en este caso crece en $150 \mathrm{MJ}$, y la exergía de los productos de fusión aumenta no tan rápidamente, lo que lleva a que sea mucho menor, en comparación con la ICP, 
TABLA III.- Balances exérgicos de la operación del horno alto con IGN

TABLE III.-Exergy balances in the blast furnace operation with NGI

\begin{tabular}{|c|c|c|c|c|c|c|c|}
\hline \multirow{2}{*}{ Índices } & \multirow{2}{*}{ Unidades } & \multicolumn{6}{|c|}{ Caudal ICP, $\mathrm{m}^{3} / \mathrm{ta}$} \\
\hline & & 0 & 55 & 80 & 110 & 140 & 175 \\
\hline Consumo de coque seco & $\mathrm{kg} / \mathrm{ta}$ & 597,7 & 555,4 & 533,0 & 509,8 & 485,8 & 461,0 \\
\hline Consumo de $\mathrm{C}$ del coque, quemado en el viento seco & $\mathrm{kg} / \mathrm{ta}$ & 348,1 & 331,2 & 322,3 & 313,0 & 303,5 & 293,6 \\
\hline Volumen gas de tragante & $\mathrm{m}^{3} / \mathrm{ta}$ & $1.923,5$ & $2.012,2$ & $2.058,7$ & $2.106,6$ & $2.155,9$ & $2.206,6$ \\
\hline Pérdidas de energía en el horno & $\mathrm{kg}$ ce/ta & 359,8 & 363,9 & 366,1 & 368,4 & 370,8 & 373,3 \\
\hline \multicolumn{8}{|l|}{ BALANCE EXÉRGICO } \\
\hline \multicolumn{8}{|l|}{ • Entradas: } \\
\hline - Exergía física del viento & $\mathrm{MJ} / \mathrm{ta}$ & $1.328,0$ & $1.377,9$ & $1.403,5$ & $1.429,4$ & $1.455,8$ & $1.482,5$ \\
\hline - Exergía física del GN & $\mathrm{MJ} / \mathrm{ta}$ & 0 & 1,5 & 2,3 & 3,2 & 4,0 & 4,9 \\
\hline - Exergía química ddel coque & $\mathrm{MJ} / \mathrm{ta}$ & $17,221,5$ & $16.002,4$ & $15.357,6$ & $14.689,1$ & $13.997,1$ & $13.281,6$ \\
\hline - Exergía química del GN & $\mathrm{MJ} / \mathrm{ta}$ & 0 & 972,4 & $3.016,1$ & $4.097,0$ & $5.215,1$ & $6.370,2$ \\
\hline - Materiales férreos & $\mathrm{MJ} / \mathrm{ta}$ & 918,1 & 918,1 & 918,1 & 918,1 & 918,1 & 918,1 \\
\hline Total & $\mathrm{MJ} / \mathrm{ta}$ & $19,490,6$ & $20,272,3$ & $20.697,1$ & $21.136,8$ & $21.590,1$ & $22.057,3$ \\
\hline \multicolumn{8}{|l|}{ - Consumos } \\
\hline \multicolumn{8}{|l|}{ - Exergía del arrabio: } \\
\hline Física & $\mathrm{MJ} / \mathrm{ta}$ & 754,6 & 754,6 & 754,6 & 754,6 & 754,6 & 754,6 \\
\hline Química & $\mathrm{MJ} / \mathrm{ta}$ & $7.957,9$ & $7.957,9$ & $7.957,9$ & $7.957,9$ & $7.957,9$ & $7.957,9$ \\
\hline \multicolumn{8}{|l|}{ - Exergía de la escoria: } \\
\hline Física & $\mathrm{MJ} / \mathrm{ta}$ & 807,5 & 807,5 & 807,5 & 807,5 & 807,5 & 807,5 \\
\hline Química & $\mathrm{MJ} / \mathrm{ta}$ & 283,7 & 283,7 & 283,7 & 283,7 & 283,7 & 283,7 \\
\hline \multicolumn{8}{|l|}{ - Exergía del gas de tragante: } \\
\hline Física & $\mathrm{MJ} / \mathrm{ta}$ & 533,2 & 610,0 & 662,9 & 725,5 & 797,7 & 879,9 \\
\hline Química & $\mathrm{MJ} / \mathrm{ta}$ & $6.366,4$ & $6.951,8$ & $7.262,6$ & $7.585,4$ & $7.920,4$ & $8.267,3$ \\
\hline Total & $\mathrm{MJ} / \mathrm{ta}$ & $16.703,3$ & $17.365,5$ & $17,729,2$ & $18.114,6$ & $18.521,8$ & $18.950,6$ \\
\hline - Pérdidas exérgicas & $\mathrm{MJ} / \mathrm{ta}$ & $2.764,3$ & $2.906,8$ & $2.968,4$ & $3.022,2$ & $3.068,3$ & $3.106,7$ \\
\hline - Perfección termodinámica del horno alto & $\%$ & 85,75 & 85,66 & 85,66 & 85,70 & 85,79 & 85,91 \\
\hline$\cdot K_{t}$ & $\%$ & 44,7 & 43,0 & 42,1 & 41,2 & 40,4 & 39,5 \\
\hline$\cdot K_{g}$ & $\%$ & 77,4 & 77,3 & 77,2 & 77,1 & 77,0 & 77,0 \\
\hline
\end{tabular}

la perfección termodinámica de los procesos que ocurren en el horno, e incluso se produce alguna disminución del $K_{g}$ en la fusión.

El análisis anterior confirma lo expuesto en (3), que el empleo de GN es una práctica cada vez menos utilizada como aporte de energía al horno alto.

\subsection{Enriquecimiento del viento con oxígeno}

El valor de la exergía del horno alto enriquecido con oxígeno, muestra que el aumento del contenido de oxígeno ocasiona mejoras en el comportamiento termodinámico y en la economía de fabricación del arrabio.

\section{CONCLUSIONES}

El análisis del balance de la exergía de la operación del horno alto, cuando se inyectan combustibles auxiliares por toberas, indica que la utilización de gas natural no es efectiva, y que la efectividad de la inyección de carbón pulverizado (ICP) crece con el aumento del caudal de inyección. Se recomienda la utilización de la ICP en combinación con el enriquecimiento del viento con oxígeno. 
TABLA IV.- Balance de exergía de la operación del horno alto con distintos contenidos de oxígeno en el viento

TABLE IV.-Exergy balances in the blast furnace operation with different oxigen contents in blast

\begin{tabular}{|c|c|c|c|c|c|}
\hline \multirow{2}{*}{ Índices } & \multirow{2}{*}{ Unidades } & \multicolumn{4}{|c|}{ Contenido de oxígeno en el viento, $\%$} \\
\hline & & 21 & 25 & 29 & 33 \\
\hline \multicolumn{6}{|l|}{ - Entradas: } \\
\hline - Exergía física del viento & $\mathrm{MJ} / \mathrm{ta}$ & $1.593,1$ & $1.359,9$ & $1.169,8$ & $1.022,9$ \\
\hline - Exergía física del GN & $\mathrm{MJ} / \mathrm{ta}$ & 2,4 & 2,4 & 2,4 & 2,4 \\
\hline - Exergía química ddel coque & $\mathrm{MJ} / \mathrm{ta}$ & $12.965,7$ & $12.965,7$ & $12.965,7$ & $12.965,7$ \\
\hline - Exergía química del GN & $\mathrm{MJ} / \mathrm{ta}$ & $3.103,3$ & $3.103,3$ & $3.103,3$ & $3.103,3$ \\
\hline - Exergía química del $\mathrm{CP}$ & $\mathrm{MJ} / \mathrm{ta}$ & $2.508,1$ & $2.508,1$ & $2.508,1$ & $2.508,1$ \\
\hline - Exergía química de los materiales férreos & $\mathrm{MJ} / \mathrm{ta}$ & 918,1 & 918,1 & 918,1 & 918,1 \\
\hline Total & $\mathrm{MJ} / \mathrm{ta}$ & $21.090,7$ & $20.857,5$ & $20.667,4$ & $20.520,5$ \\
\hline \multicolumn{6}{|l|}{ - Consumos } \\
\hline \multicolumn{6}{|l|}{ - Exergía del arrabio: } \\
\hline Física & $\mathrm{MJ} / \mathrm{ta}$ & 754,6 & 754,6 & 754,6 & 754,6 \\
\hline Química & $\mathrm{MJ} / \mathrm{ta}$ & $7.957,9$ & $7.957,9$ & $7.957,9$ & $7.957,9$ \\
\hline \multicolumn{6}{|l|}{ - Exergía de la escoria: } \\
\hline Física & $\mathrm{MJ} / \mathrm{ta}$ & 807,5 & 807,5 & 807,5 & 807,5 \\
\hline Química & $\mathrm{MJ} / \mathrm{ta}$ & 283,7 & 283,7 & 283,7 & 283,7 \\
\hline \multicolumn{6}{|l|}{ - Exergía del gas de tragante: } \\
\hline Física & $\mathrm{MJ} / \mathrm{ta}$ & 930,7 & 738,0 & 591,6 & 491,4 \\
\hline Química & $\mathrm{MJ} / \mathrm{ta}$ & $7.843,1$ & $7.815,0$ & $7.795,4$ & $7.784,4$ \\
\hline Total de consumo & $\mathrm{MJ} / \mathrm{ta}$ & $18.577,5$ & $18.356,6$ & $18.190,6$ & $18.079,4$ \\
\hline - Pérdidas exérgicas & $\mathrm{MJ} / \mathrm{ta}$ & $2.513,2$ & $2.500,9$ & $2.476,8$ & $2.441,1$ \\
\hline - Perfección termodinámica del horno alto & $\%$ & 88,08 & 88,10 & 88,11 & 88,18 \\
\hline • $K_{t}$ & $\%$ & 41,31 & 41,77 & 42,16 & 42,46 \\
\hline - $K_{g}$ & $\%$ & 78,50 & 79,37 & 80,10 & 80,68 \\
\hline
\end{tabular}

\section{REFERENCIAS}

(1) Shargut, Ya. y Petela, R. Exergy. Moscú, Energy. 1968. 276 págs. (en ruso).

(2) Stepanov, V.S. Analysis of energetical perfection of technological processes. Novosibirsk, Nauka, 1984. 273 págs. (en ruso).
(3) Borodulin, A.V., Kovtun, A.F. y Henkina, O.E. Isv. Vyssh. Chernaya Metall., 8, 1990: 104-110.

(4) Rossard, C. y Blain, P. Mem. Sci. Rev. Metall., LVI (3), 1959: 285-300.

(5) Luton, M.J. y Sellars, C.M. Acta Metall., 17, 1969: 1.033-1.043.

(6) Babich, A., Garcia, L., Formoso, A., Mochon, J. y Cores, A. Rev. Metal. Madrid, 33 (2), 1997: 71-79. 